\title{
Auftakttreffen der neugegründeten Arbeitsgruppe „Feministische Perspektiven in der Medizin- und Bioethik" in der AEM
}

\author{
Online, 21. September 2021
}

\section{Maximiliane Hädicke}

Angenommen: 8. November 2021 / Online publiziert: 18. November 2021

(C) Der/die Autor(en) 2021

Beim Online-Auftakttreffen der neugegründeten Arbeitsgruppe Feministische Perspektiven in der Medizin- und Bioethik (FME) am 21. September 2021 ließen sich die Zoom-Kacheln nicht mehr auf einer Seite abbilden. Nach und nach füllte sich der Bildschirm mit insgesamt 35 Gesichtern voller Tatendrang. Ein Grund für das rege Interesse war vermutlich, dass feministische Perspektiven aus den Debatten der internationalen Medizin- und Bioethik kaum wegzudenken sind, es in der deutschsprachigen Medizin- und Bioethik bisher jedoch keine eigene Plattform für sie gab. Dies soll sich nun ändern. Koordiniert wird die Arbeitsgruppe von Regina Müller (Tübingen), Dr.in Mirjam Faissner (Bochum), Dr.in Kris Vera Hartmann (Heidelberg), Dr.in Isabella Marcinski-Michel (Berlin/Göttingen) und Dr.in Merle Weßel (Oldenburg). Die Ziele der Auftaktveranstaltung waren, den Teilnehmenden die Gelegenheit zu bieten, sich gegenseitig kennenzulernen, sich über die Erwartungen an die Arbeitsgruppe zu verständigen und mögliche Perspektiven der Zusammenarbeit auszuloten.

In Erinnerung bleiben nicht nur die gute Vorbereitung und die interaktive Umsetzung der Veranstaltung, sondern auch erste, konstruktive Ergebnisse.

\section{Wie passen Feminismus und Medizinethik zusammen?}

Für das Logo der Arbeitsgruppe haben die Koordinatorinnen sicherlich nicht zufällig die Farbe Lila gewählt. Sie wird verwendet, um feministische Aktionen als solche zu markieren. Doch wie passt die soziale Bewegung zur akademischen Disziplin

\footnotetext{
Maximiliane Hädicke, M. mel. ( $\square)$

Institut für Ethik und Geschichte der Medizin, Universitätsmedizin Göttingen,

Humboldtallee 36, 37073 Göttingen, Deutschland

E-Mail: maximiliane.haedicke@med.uni-goettingen.de
} 
der Medizinethik? Antworten auf diese Frage gibt es unterschiedliche. Einige präsentierte Regina Müller, die Initiatorin der Arbeitsgruppe, den Teilnehmer_innen in einem kurzen einleitenden Vortrag. Dabei identifizierte sie sowohl thematische als auch methodische Überschneidungen.

So präsentierte sie, dass Fragen der Gerechtigkeit ein klassisches Themenfeld der Medizinethik ausmachen können. Sie würden etwa dann diskutiert, wenn über die faire Verteilung knapper Ressourcen im Gesundheitswesen verhandelt werde. Aktuell sei hierbei die Tendenz zu beobachten, dass solche Fragen vermehrt im Lichte gesellschaftlicher Vielfalt debattiert würden. Eine methodische Entwicklung in der Medizinethik sei, dass Stakeholder zunehmend mehr an öffentlichen Debatten teilnehmen und in empirisch-ethischen Forschungsprojekten eingebunden seien. Dies würde von einigen Stimmen im Fach als Zugewinn für medizinethische Erkenntnisprozesse erachtet.

Sowohl diese thematischen als auch die methodischen Entwicklungen ließen sich als Schnittstellen zu feministischen Bewegungen interpretieren. Diese kämpfen zum einen seit jeher für gerechtere gesellschaftliche Verhältnisse und gehen dabei zum anderen partizipativ vor. Partizipation sei für feministische Ansätze insbesondere deshalb charakteristisch, weil sie das Ziel verfolgen, den (von Ungerechtigkeit) betroffenen Subjekten Gehör zu verschaffen und ihre Sichtweisen in den Mittelpunkt zu stellen.

Doch der progressive Anspruch feministischer Bewegungen geht über diese beiden Aspekte hinaus. Zentral sei, dass sie Machtverhältnisse hinterfragen und oftmals verdeckte, benachteiligende soziale Strukturen offenlegen, um auf diese Weise eine nachhaltige Grundlage für gesellschaftliche Veränderung zu schaffen. Es könne sich, so Regina Müller, wiederum als Zugewinn für die medizinethischen Debatten erweisen, machtkritische Analysen stärker in die gängige Praxis des Fachs zu integrieren.

Im Wesentlichen kristallisierten die Koordinatorinnen fünf mögliche Ebenen einer feministisch praktizierten Medizinethik in Deutschland heraus. Sie könnte:

- sich verstärkt bestimmten angewandten medizinethischen Problemstellungen zuwenden, beispielsweise der Benachteiligungen von Schwarzen Frauen* im Gesundheitswesen.

- das Spektrum der theoretischen Zugänge erweitern, etwa durch einen intersektionalen Blick auf die soziale Position von Individuen.

- die Wahrnehmung erweitern, zum Beispiel durch die Problematisierung geschlechtsbasierter Datenlücken in der medizinischen Forschung.

- die Implikationen zum Beispiel dieser Datenlücken für die medizinische Praxis systematisch analysieren.

- einen feministischen Blick auf die Medizinethik als akademische Disziplin richten und beispielsweise untersuchen, inwiefern Diversität an den deutschen GTE-Instituten abgebildet wird.

Es wurde vorgeschlagen, diese fünf Ebenen als erste Strukturierungs- und Orientierungshilfe für die zukünftige Zusammenarbeit zu nutzen. 


\section{Ergebnisse des ersten Treffens}

In Vorbereitung auf das Treffen haben die Koordinatorinnen die Interessen und fachlichen Hintergründe der Teilnehmer_innen bereits in einer kleinen Online-Umfrage erfasst. Im Auftakttreffen präsentierten sie die Ergebnisse in einer anschaulichen Übersicht und kombinierten dies mit interaktiven Elementen, was das Kennenlernen erleichterte.

Zudem führte die gute Vorbereitung des Auftakttreffens dazu, dass die erste gemeinsame Zeit schon für die inhaltliche Arbeit genutzt werden konnte. In themenspezifischen Kleingruppen wurde sich vertiefend über Interessen ausgetauscht, Vorarbeiten zusammengetragen und Zukunftsvisionen entwickelt. Insgesamt vier Kleingruppen befassten sich mit den Themen: a) Methoden und Lehre, b) Intersektionalität und Queertheory, c) Digitalisierung sowie d) Reproduktionsmedizin. Die Ergebnisse wurden dokumentiert und sollen den weiteren Treffen als Arbeitsgrundlage dienen.

Es überraschte wenig, dass es den anwesenden Teilnehmer_innen nicht an geteilten Interessen mangelte. Aus der Vielfalt lassen sich an dieser Stelle nur einige exemplarisch nennen. So hielt die Untergruppe Methoden und Lehre etwa fest, dass sie sich darüber austauschen möchte, wie sich feministische Perspektiven konkret in die GTE-Lehre und in die medizinethische Forschung einbringen ließen.

In der Untergruppe Intersektionalität und Queertheory wurde sich u. a. darauf verständigt, dass sich der systematischen Reflexion von Positionalität im medizinethischen Forschungsprozess zugewandt werden könne.

Im Themenfeld Digitalisierung ist es ein geteiltes Interesse der Teilnehmer_innen mit Konzepten der relationalen Autonomie zu arbeiten. In der Gruppe Reproduktionsmedizin wurde sich u. a. auf die Anliegen verständigt, sich mit Fragen epistemischer Ungerechtigkeit zwischen Ärzt_innen und Patient_innen zu befassen und Machtverhältnisse zu analysieren, etwa am Beispiel von Gewalt unter der Geburt. In allen Kleingruppen konnten zudem einige relevante Vorarbeiten der Teilnehmer_innen zusammengetragen werden. Diese reichen von thematisch oder methodisch relevanten Publikationen über vorhandene Netzwerkstrukturen bis hin zu politischem Engagement.

\section{Ausblick}

Im Auftakttreffen der AG für feministische Perspektiven in der Medizin- und Bioethik war eine kreative Energie spürbar, wie sonst nur selten in Online-Veranstaltungen dieser Größe. Am Ende der Veranstaltung schien klar zu sein, dass ein großes Bedürfnis zu weiterem Austausch besteht. Zunächst sollen die Untergruppen inhaltlich weiter konkretisiert und eine Zukunftsvision der Gesamtgruppe entwickelt werden.

Fest steht zu diesem Zeitpunkt auch, dass vielfältige Wege für die weitere Entwicklung dieser jungen Arbeitsgruppe offenstehen. Es sind Ideen gefallen wie die gemeinsame Planung von Veranstaltungen oder die gemeinsame Entwicklung von 
Forschungsideen. Ob themenspezifische, politische Positionierungen veröffentlicht werden sollen, ist ebenfalls noch offen.

Bei Interesse an der Mitarbeit in der Arbeitsgruppe können Sie sich per E-Mail an Regina Müller wenden (regina.mueller@uni-tuebingen.de).

Funding Open Access funding enabled and organized by Projekt DEAL.

Open Access Dieser Artikel wird unter der Creative Commons Namensnennung 4.0 International Lizenz veröffentlicht, welche die Nutzung, Vervielfältigung, Bearbeitung, Verbreitung und Wiedergabe in jeglichem Medium und Format erlaubt, sofern Sie den/die ursprünglichen Autor(en) und die Quelle ordnungsgemäß nennen, einen Link zur Creative Commons Lizenz beifügen und angeben, ob Änderungen vorgenommen wurden.

Die in diesem Artikel enthaltenen Bilder und sonstiges Drittmaterial unterliegen ebenfalls der genannten Creative Commons Lizenz, sofern sich aus der Abbildungslegende nichts anderes ergibt. Sofern das betreffende Material nicht unter der genannten Creative Commons Lizenz steht und die betreffende Handlung nicht nach gesetzlichen Vorschriften erlaubt ist, ist für die oben aufgeführten Weiterverwendungen des Materials die Einwilligung des jeweiligen Rechteinhabers einzuholen.

Weitere Details zur Lizenz entnehmen Sie bitte der Lizenzinformation auf http://creativecommons.org/ licenses/by/4.0/deed.de.

\section{Einhaltung ethischer Richtlinien}

Interessenkonflikt M. Hädicke gibt an, dass kein Interessenkonflikt besteht.

Ethische Standards Für diesen Beitrag wurden von der Autorin keine Studien an Menschen oder Tieren durchgeführt. Für die aufgeführten Studien gelten die jeweils dort angegebenen ethischen Richtlinien. 